\title{
Conhecimento Financeiro em Estudantes Universitários: análise pela Teoria de Resposta ao Item.
}

\section{Guilherme Santos Souza ${ }^{1}$}

UFU, Universidade Federal de Uberlândia, Faculdade de Gestão e Negócios (FAGEN), Uberlândia, MG, Brasil.

Pedro Paulo Melo Arantes ${ }^{2}$ iD

UFU, Universidade Federal de Uberlândia, Faculdade de Gestão e Negócios (FAGEN), Uberlândia, MG, Brasil.

\section{Pablo Rogers 3}

UFU, Universidade Federal de Uberlândia, Faculdade de Gestão e Negócios (FAGEN), Uberlândia, MG, Brasil.

\section{Dany Rogers ${ }^{4}$}

UFU, Universidade Federal de Uberlândia, Faculdade de Administração, Ciências Contábeis,

Engenharia de Produção e Serviço Social (FACES), Ituiutaba, MG, Brasil.

\begin{abstract}
Resumo
A recente democratização do crédito tem permitido a população um aumento em seu padrão de consumo, no entanto, o nível de conhecimento financeiro não acompanhou tal crescimento, sendo isso um gatilho para sérios problemas financeiros. O presente estudo teve como objetivo medir o nível de conhecimento financeiro em estudantes universitários, verificando se existe diferença entre calouros e egressos, e, além disso, analisar se existe alguma diferença no domínio do tema entre diversos cursos de graduação como engenharias, de negócios e de humanas (i.e entre alunos com níveis diferentes de educação matemática). Para isso, foi aplicado um questionário que foi refinado utilizando a Teoria de Resposta ao Item (TRI), validada pelo teste DIF (Differential Item Functioning). A amostra contou com 232 estudantes e os resultados concluíram que existe diferença no conhecimento financeiro entre os grupos, sendo maior em estudantes do último ano e em alunos dos cursos de engenharias e de negócios e entre àqueles que possuem uma educação matemática mais sólida, o que pode indicar que a matemática ajuda para uma melhor alfabetização financeira do indivíduo. Adicionalmente, também foram encontradas diferenças de conhecimento financeiro por gênero, raças/etnias, renda e escolaridade dos pais.
\end{abstract}

Palavras-chave: Conhecimento Financeiro; Alfabetização Financeira; Teoria de Resposta ao Item.

\footnotetext{
${ }^{1}$ Mestre em Administração pela FAGEN-UFU. E-mail: guilhermessantos042@ gmail.com

${ }^{2}$ Mestre em Administração pela FAGEN-UFU. E-mail: pedropauloadm@yahoo.com.br

${ }^{3}$ Doutor em Administração pela FEA-UFU. Professor de Finanças da FAGEN-UFU. E-mail: pablorogers@ufu.br

${ }^{4}$ Doutor em Administração pela EAESP-FGV. Professor de Finanças da FACES-UFU e Coordenador do Núcleo de Educação Financeira (NEF) da UFU. E-mail: danyrogers@ufu.br
} 


\title{
Financial knowledge in university students: analysis by Item Response Theory
}

\begin{abstract}
The recent democratization of credit has allowed the population to increase its consumption pattern, however, the level of financial knowledge has not kept up with this growth, which is a trigger for serious financial problems. The present study aimed to measure the level of financial knowledge in university students verifying if there is a difference between incoming students and graduates and in addition to analyze if there is any difference in the domain of the theme between several undergraduate courses such as engineering, business and humanities (i.e. among students of courses with different levels of mathematical education). For this a questionnaire was applied and refined using the Item Response Theory (IRT), validated by the Differential Item Functioning (DIF) test. The sample had 232 students and the results concluded that there is a difference in financial knowledge between the groups, being higher in students of the last year and in students of engineering and business courses, to those who have a less dense mathematical education, which may indicate that mathematics helps for better financial literacy of the individual. Additionally, differences in financial knowledge by gender, races/ethnicities, income and parental education were also found.
\end{abstract}

Keywords: Financial Knowledge; Financial Literacy; Item Response Theory.

\section{Conocimiento financiero em estudiantes universitarios: análisis por Teoría de Respuesta al Ítem}

\section{Resumen}

La reciente democratización del crédito ha permitido que la población incremente su patrón de consumo, sin embargo, el nivel de conocimiento financiero no se ha mantenido a la par de este crecimiento, lo que es un detonante de serios problemas financieros. El presente estudio tuvo como objetivo medir el nivel de conocimiento financiero en estudiantes universitarios comprobando si hay alguna diferencia entre estudiantes entrantes y egresados y además analizar si existe una diferencia en el dominio del tema entre varios cursos de pregrado como ingeniería, negocios y humanidades (es decir, entre estudiantes de cursos con diferentes niveles de educación matemática). Para ello se aplicó y perfeccionó un cuestionario utilizando la Teoría de Respuesta al Ítem (TRI), validado por la prueba de Funcionamiento Diferencial del Ítem (FDI). La muestra tuvo con 232 estudiantes y los resultados concluyeron que hay una diferencia en el conocimiento financiero entre los grupos, siendo mayor en los estudiantes del último año y en los estudiantes de las carreras de ingeniería y negocios, a los que tienen una educación matemática menos densa, lo que puede indicar que las matemáticas ayudan a mejorar la educación financiera del individuo. Además, también se encontraron diferencias en el conocimiento financiero por género, razas / etnias, ingresos y educación de los padres.

Palabras clave: Conocimiento Financiero; Educación Financiera; Teoría de la Respuesta al Ítem.

\section{Introdução}

A expansão do crédito no Brasil vem ocorrendo de forma exponencial, mas o entendimento dos indivíduos acerca de como melhor utilizar esse novo poder de compra obtido por meio destes recursos financeiros, seja na forma de cartões de crédito ou empréstimos, não acompanharam esse crescimento (SOUZA FERNANDES e CANDIDO, 2014). Esse crescimento do crédito trouxe 
principalmente para àqueles que até então não tinham acesso, maiores facilidades para a adesão de produtos financeiros (ARCEO-GÓMEZ e VILLAGÓMEZ, 2017). Mas essa situação vem acarretando um alto nível de endividamento, bem como um preocupante índice de inadimplência das famílias, em especial em faixas de renda mais baixas, que estão mais vulneráveis financeiramente. Assim, conseguir alcançar ou realizar sonhos de consumo utilizando esses recursos pode acabar se tornando um pesadelo quando a falta de controle e o conhecimento financeiro resultam no acúmulo de dívidas (FRANCISCHETTI et. al, 2016).

Nesse contexto, a habilidade de gerenciar as finanças pessoais têm se tornado cada vez mais necessária frente aos cenários futuros incertos e uma economia dinâmica, além dessa maior disponibilização de crédito que exige um maior entendimento da utilização do dinheiro para não incorrer em situações de endividamento. Quanto maior a habilidade com o dinheiro e o conhecimento financeiro, mais o indivíduo possui ferramentas para lhe auxiliar em suas tomadas de decisões, aumentando seu bem-estar e evitando o endividamento (KLAPPER, LUSARDI e PANOS, 2013).

Estudos como Donadio, Campanario e Rangel (2012) buscaram entender sobre os fatores que influenciam no nível de endividamento pessoal e indicaram que a falta de conhecimento de financeiro, bem como um melhor comportamento e atitude para lidar com as próprias finanças (atitude e comportamento financeiro), culminaram para o seu aumento. Nesse sentido, uma boa alfabetização financeira, que contempla conhecimento, comportamento e atitude financeira, conforme OCDE (2016), seria um item determinante para o endividamento do indivíduo.

Notoriamente, entre os fatores que compõem a alfabetização financeira, o conhecimento é o mais difundido e mais possível de ser trabalhado, pois se trata de um processo de capacitação individual, que visa o desenvolvimento de habilidades para uma tomada de decisão mais assertiva com relação a forma de lidar com o dinheiro em um contexto pessoal (POTRICH, VIEIRA e KIRCH, 2014; METTE e MATOS, 2016).

Diversos estudos ao buscar entender se as pessoas possuem bom controle de suas ações financeiras ou se possuem conhecimento financeiro para lhe direcionar frente à alguma tomada de decisão mostraram um cenário preocupante, onde o grau de entendimento sobre conhecimento financeiro é baixo (CHEN e VOLPE, 1998; POTRICH, VIEIRA e CERETA, 2013; SCHERESBERG, 2013; GROHMANN, KOUWENBERG e MENKHOFF, 2015; POTRICH et al., 2015b; POTRICH et al., 2015a; OCDE, 2016; ARCEO-GÓMEZ e VILLAGÓMEZ, 2017). Estes estudos mostram existir uma correlação positiva entre escolaridade e o nível de entendimento acerca do bom uso do dinheiro. No entanto, mesmo entre pessoas com um bom nível de escolaridade como estudantes universitários, o conhecimento sobre esse assunto e o comportamento do indivíduo em relação às finanças pessoais ainda é limitado. 
Sendo uma habilidade que quanto mais cedo desenvolvida melhor, dada a complexidade que se desenvolve na vida do jovem à medida que avança na idade, principalmente na fase da adolescência (LUSARDI, MITCHELL e CURTO, 2010; SOHN et al., 2012; ARCEO-GÓMEZ e VILLAGÓMEZ, 2017), que compreende também os estudantes universitários, um melhor conhecimento sobre este público se faz necessário.

Assim, dada a situação que pessoas com maior escolaridade tendem a apresentar maiores conhecimentos financeiros, mas que mesmo assim pesquisas com estudantes universitários têm apresentado resultados insatisfatórios, será que a vivência acadêmica proporciona ao estudante situações ou oportunidades que lhe ajudam na sua evolução do conhecimento/entendimento/educação financeira ao longo do curso escolhido? Será que existe diferença para essa evolução do conhecimento financeiro dependendo do curso realizado pelo estudante, em especial, entre cursos com maior foco em matemática versus cursos mais relacionados às humanas?

Sabendo então da importância deste tema relacionado a alfabetização financeira, que a graduação é um marco na vida e uma preparação do indivíduo para o mercado de trabalho e para a vida, e, reforçando a importância de um bom conhecimento financeiro para diversos aspectos pessoais, sociais e inclusive de saúde de qualquer indivíduo, cabe o questionamento se a vivência acadêmica também melhora o grau de conhecimento financeiro dos indivíduos. Nesse sentido, este estudo se propõe a mensurar o grau de conhecimento financeiro em estudantes universitários, verificando se existem diferenças entre alunos ingressantes e concluintes e entre cursos de graduação, em especial, entre cursos mais relacionados às ciências exatas e ciências humanas.

\section{Revisão de Literatura}

Com relação ao conceito de educação financeira, não existe um padrão consensual entre os estudiosos sobre o tema, sendo muito confundido e utilizado como sinônimo para alfabetização financeira. Sabe-se que a literatura trata conhecimento financeiro como uma das bases da alfabetização financeira, e, por isso, Huston (2010), buscando um padrão, define que a alfabetização financeira extrapola o fato de o indivíduo possuir o conhecimento ou a educação financeira, considerando também que ele deve ser capaz de utilizar esse conhecimento, aplicando-os em suas finanças pessoais.

Em 2010, o tema alfabetização financeira começou a ser desenvolvido por entidades governamentais no Brasil por iniciativa do governo federal juntamente com outros órgãos públicos e algumas instituições privadas, tendo assim o advento da Estratégia Nacional de Educação Financeira - ENEF. Na ENEF a educação financeira é entendida como um tema transversal, que dialoga com as mais diversas disciplinas do currículo do Ensino Fundamental e Médio, possibilitando aos alunos 
compreender como concretizar suas aspirações e estar preparado para as diversas fases da vida. Além de colaborar para as áreas de conhecimento da educação financeira, as iniciativas da ENEF para os ensinos fundamentais e médio também são pensadas para proporcionar a melhoria de desempenho dos alunos em Língua Portuguesa e Matemática (ENEF, 2021).

Mas antes de ganhar notoriedade mundial e ser instituído como uma política de estado no Brasil, pesquisas sobre conhecimento, comportamento e atitudes financeiras (alfabetização financeira) já eram desenvolvidas em diversos países. Nos Estados Unidos, Chen e Volpe (1998) entrevistaram 924 graduandos em 14 universidades e concluíram que esse público precisava aprimorar suas noções de como gerir suas finanças pessoais de forma a tomarem decisões mais adequadas.

Um pouco mais tarde, Beal e Delpachita (2003) também concluíram que alunos universitários na Austrália apresentavam baixo nível de alfabetização financeira, principalmente com relação aos conhecimentos financeiros. Como possíveis causas para essa situação os autores citaram, principalmente, a falta de ensino de habilidades financeiras em escolas secundárias. Além disso, os resultados sugeriram que esse tema se torna mais importante para as pessoas à medida que aumenta a renda e a experiência profissional, sinal de que os indivíduos buscam aprimorar seus conhecimentos financeiros a fim de melhor gerir seus recursos, mas em geral, isso acontecia por meio de tentativa e erro (o indivíduo aprendia mais pela vivência do que por uma busca por aprimorar os conhecimentos e habilidades financeiras).

Estudos relacionados a alfabetização financeira não são triviais pois diversos fatores sociais ou familiares têm participação significativa em como o indivíduo aprende a lidar com o dinheiro, desde a infância e/ou adolescência. Cunha e Heckman (2007) desenvolveram um modelo teórico que mostra o vínculo entre os antecedentes familiares (i.e. educação e capacidade cognitiva) com o comportamento e os resultados alcançados pelas pessoas em suas vidas mais tarde.

Seguindo essa linha, da importância e influência que os pais exercem na formação do conhecimento e comportamento dos filhos, Grohmann, Kouwenberg e Menkhoff (2015) exemplificam que os pais com alto nível de escolaridade e/ou que possuem hábitos de controle de gastos e de investimentos, influenciam de forma positiva na formação cognitiva da criança bem como em sua alfabetização financeira. No entanto, estes fatores familiares e sociais não devem ser determinantes: uma pessoa deve ser capaz de lidar com suas finanças dentro do seu contexto, se adequando à sua própria realidade.

Considerando que na fase adulta a vida financeira de uma pessoa torna-se cada vez mais complexa, a idade ideal para se ter contato com esses conceitos mais naturalmente, de modo a incorporá-los no seu dia a dia, é na adolescência um vez que neste momento os adolescentes 
costumam lidar diretamente com dinheiro, começam a ter alguma fonte de renda, iniciada muitas vezes por meio de mesadas, vínculo empregatício relacionado a programas de menor aprendiz, participação de projetos com bolsa-auxílio, e quando na faculdade, até mesmo já se inserindo no mercado de trabalho (ARCEO-GÓMEZ e VILLAGÓMEZ, 2017).

O tema alfabetização financeira apresenta importância significativa quando referenciada aos estudantes universitários, justamente dada a complexidade inerente ao momento na vida dos jovens. Pois quando não saem de casa para estudarem em outras cidades, eles passam a necessitar de alguma fonte de renda, para, entre outras coisas, viabilizarem sua participação em congressos, cursos, palestras ou até mesmo a compra de algum equipamento que o curso exija ou que lhes auxiliem nos seus estudos.

Nesse sentido, as pesquisas mostram que mesmo com o apoio e interesse de uma organização internacional como a OCDE e com ações multisetoriais e o apoio do governo federal no Brasil, o grau de entendimento sobre o assunto, principalmente quando o público-alvo são estudantes universitários, ainda é baixo. Prova disso são os resultados obtidos por Norvilitis et al. (2006) ao entrevistarem 448 alunos de cinco universidades nos Estados Unidos sobre o que ocasionava o alto endividamento deles, concluindo ser o principal motivo o baixo conhecimento financeiro.

Nano e Polo (2016) verificando se o aumento dos estudos afeta o grau de conhecimento do indivíduo incluíram na sua amostra estudantes tanto de graduação quanto de mestrado, entrevistando um total de 607 alunos de diversos cursos na Albânia. A conclusão do trabalho mostra que apesar dos estudantes de mestrado possuírem mais conhecimento financeiro que os alunos de graduação, no geral, ambos os grupos apresentaram um baixo entendimento sobre o assunto, principalmente quando verificado juntamente o comportamento e a atitude financeira (alfabetização financeira).

No Brasil, o cenário não é menos preocupante. Potrich, Vieira e Cereta (2013) criaram uma escala de alfabetização financeira e mediram o grau de conhecimento financeiro em 534 alunos de cursos universitários que ofereciam conteúdos de matemática, matemática financeira e gestão financeira na cidade de Santa Maria - Rio Grande do Sul. Concluíram que esses estudantes não apresentaram níveis desejáveis de alfabetização financeira, visto o resultado insatisfatório de conhecimento e compreensão de questões financeiras.

Nascimento et al. (2016), utilizando a Teoria de Resposta ao Item (TRI), mediram o grau de alfabetização financeira em 307 estudantes de Administração de Empresas e concluíram que mesmo o currículo desse curso possuindo conteúdos de cunho financeiro e matemática, o grau de alfabetização financeira dos estudantes, principalmente na questão do conhecimento financeiro, foi baixo e preocupante. 
Pelo que se percebe, mesmo os cursos que apresentam em seu currículo conteúdos de gestão financeira ou matemática não se mostraram determinantes para o indivíduo apresentar uma alfabetização financeira satisfatória. Contudo, como uma forma de melhorar esse cenário, Potrich, Vieira e Cereta (2013) reforçam a necessidade de desenvolver ações efetivas para minimizar o problema do analfabetismo financeiro, sugerindo a inclusão de disciplinas de gestão financeira pessoal e noções de finanças de mercado em todos os cursos de graduação, independente da área de ensino. Bemel et al. (2016) reforçaram esse argumento ao sugerirem que as instituições de ensino deveriam ser as responsáveis por oferecer conhecimentos financeiros básicos para os alunos.

Quando analisados cursos específicos de finanças pessoais, Mandell (2009) não encontrou evidências fortes de que indivíduos que faziam esse tipo de curso apresentaram uma melhora na sua alfabetização financeira. Adicionalmente, Norvilitis et al. (2006) e depois Jacob (2016) sugeririam soluções multifacetas, não focando apenas a disponibilização de cursos para ensinar a educação financeira, mas também incentivo de mudanças de comportamento e atitude financeira.

Não somente a relação do indivíduo com sua renda (pagar contas, prioridades de consumo, poupança e aposentadoria) são motivos de preocupação de pesquisadores. Além da relação do indivíduo com o dinheiro, e os impactos que isso causa na sua saúde financeira, estudos também verificaram como essa dicotomia impacta em outras questões como saúde fisiológica e psicológica e nível de poupança.

Bemel et al. (2016) encontraram que a saúde financeira causa impacto direto, de uma forma ou de outra, nas seis dimensões da saúde consideradas pela medicina: saúde física, emocional, intelectual, espiritual, social e com relação a como o corpo reage às influências do ambiente externo. Verificaram as reações causadas em estudantes universitários em alguma dimensão da saúde, quando considerados aspectos relacionados ao conhecimento financeiro e boas práticas de gestão de suas finanças pessoais, verificando que quando a saúde financeira deles estavam ruins, algum aspecto da sua saúde também era afetado, principalmente a psicológica.

\section{Metodologia}

O instrumento utilizado para medir o grau de conhecimento financeiro dos entrevistados foi adaptado das pesquisas realizadas por Potrich, Vieira e Cereta (2013) e Potrich et al. (2015a). Com relação ao questionário aplicado, os itens de sua versão final demandam conhecimentos sobre juros e poupança, valor do dinheiro no tempo, inflação, ativos financeiros e de risco.

Para medir o grau de conhecimento financeiro dos estudantes foi realizada a análise por meio da Teoria da Resposta ao Item - TRI, pois em detrimento da Teoria Clássica dos Testes - TCT, quando a amostra é composta por poucas observações, a TRI se mostra mais adequada (i.e. para conseguir uma melhor confiabilidade, validade e erros padrões pela TCT se faz necessário muitos 
participantes) (EMBRETSON, 1996; REISE, AINSWORTH e HAVILAND, 2005; RECKASE, 2009). Além disso, a análise pela TRI tem a possibilidade de trazer benefícios com relação a comparabilidade de desempenho de alunos longitudinalmente e por meio de estudos, populações, realidades e programas distintos (NASCIMENTO et al., 2016).

Da amostra inicial de 271 alunos, 39 questionários foram descartados devido ao nãopreenchimento de algumas questões, assim, foram consideradas 232 observações. Devido a importância do conhecimento financeiro para uma boa gestão das finanças pessoais, sendo esta necessária para qualquer pessoa que possua renda, o questionário foi aplicado aos diferentes cursos, alguns destes ofertando em sua grade curricular disciplinas específicas de finanças, matemática e gestão financeira. Os cursos que os alunos foram entrevistados foram: Pedagogia, Administração, Economia, Ciências Contábeis, Relações Internacionais e Engenharias. Para verificar se existiu variação (positiva ou negativa) entre alunos ingressantes e concluintes foram entrevistados alunos do primeiro e último ano da graduação, considerando assim a conclusão por parte destes alunos de disciplinas relacionados à tema específico sobre conhecimento financeiro como matemática e finanças de maneira geral.

Com relação a estimação dos parâmetros pela TRI, verificou-se que o modelo mais adequado para medir o nível de conhecimento financeiro dos discentes foi o modelo logístico unidimensional para itens dicotômicos com dois parâmetros (2PLM), por ser mais consistente para essa função visto a categoria binária das respostas (correta e incorreta). Este modelo está alinhado com os estudos de Nascimento et al. (2016) e Knoll e Houts (2012).

Os parâmetros estimados mensuram o nível de discriminação de cada questão, sendo que para esse estudo distingue-se os discentes com níveis de conhecimento financeiro, adicionalmente refletem a inclinação da curva do modelo logístico (i.e. quanto maior o parâmetro, mais robusta é a capacidade de discriminação dos alunos com níveis diferenciados de conhecimento financeiro). E o parâmetro dificuldade que tem a capacidade de avaliar se um item se comporta de maneira diferente entre os respondentes com o mesmo valor de traço latente (TERESI, 2006), no caso, verificou se os indivíduos com mesmo nível de traços latentes de conhecimento financeiro apresentaram diferentes probabilidades de selecionar uma mesma resposta (EDELEN et al., 2006; TERESI, 2006).

Para a verificação da existência de diferenças entre as respostas dos alunos dos mais diferentes grupos de pesquisa foram realizados os testes Mann-Whitney $(Z)$, teste de Kruskal-Wallis $\left(\chi^{2}\right)$ e a Correlação de Spearman $(\rho)$. O teste Mann-Whitney verifica a hipótese nula de que duas amostras independentes são de populações com mesmas distribuições, enquanto o teste Kruskal-Wallis verifica a hipótese de que três ou mais amostras possuem as mesmas distribuições. A Correlação de Spearman ( $\rho$ ) mensura o grau de associação entre duas variáveis ordinais. 


\section{Resultados}

\subsection{Análise descritiva e perfis}

Ao analisar o perfil dos respondentes verifica-se que a maioria é do sexo masculino $(53,45 \%)$, com idade média de 21 anos, com renda familiar acima de $\mathrm{R} \$ 5.800,00$ (34,05\%), sem dependentes $(93,53 \%)$, não trabalha $(70,69 \%)$ e se autodeclara branco $(69,83 \%)$. O percentual de pai e mãe com ensino médio são, respectivamente, $34,91 \%$ e $52,16 \%$, e os pais que possuem ensino superior são de 29,74\% para pai e 31,9\% para a mãe. Com relação ao curso, a maioria dos respondentes são de engenharias $(50,43 \%)$, seguidos dos cursos de negócios $(35,78 \%)$ e de humanas $(13,79 \%)$, dividido entre $57,76 \%$ de alunos do primeiro e $42,24 \%$ do último ano.

Após conhecer o perfil sociodemográfico dos respondentes buscou-se compreender os resultados obtidos nas questões de conhecimento financeiro. Constatou-se que $31 \%$ dos respondentes não entendem sobre o funcionamento da remuneração da poupança e $28 \%$ mostraram dificuldades em entender conceitos de juros quando relacionados a inflação, permitindo concluir que mesmo sendo a opção de investimento mais simples e segura, ainda não é algo totalmente claro para esse público.

No que diz respeito a questão que abordava sobre o conceito da perda do poder aquisitivo do dinheiro ao longo do tempo $37 \%$ dos respondentes acertaram, em contrapartida, a maioria mostrou conhecer sobre os impactos causados pela inflação no poder de consumo (79\%) e nos custos de vida e subsistência (92\%). É possível perceber que existe algum entendimento sobre o conceito de inflação, apesar de não conseguirem vinculá-lo ao valor do dinheiro no tempo.

Quando questionados sobre conceitos de riscos, a maioria mostrou entender sobre estratégias de mitigação (82\%) e sua relação linear com retornos (73\%). Apesar disso, nas questões quanto ao retorno das ações no longo prazo, a minoria conseguiu equacionar o conceito de quanto maior o risco maior o retorno (34\%) usualmente atribuído as ações, apesar de a maioria a considerar como o ativo de maior oscilação ao longo do tempo (78\%), ou seja, eles entendem que é um ativo arriscado.

Sobre juros, quando a noção é para a avaliação de recursos de terceiros (empréstimos) o nível de respostas corretas diminuem, um total de $60 \%$ acertaram sobre a relação do pagamento de juros ao longo do período de um empréstimo e $65 \%$ sobre o cálculo da taxa de juros. As questões com mais acertos foram aquelas relacionadas a divisão de valores (98\%) e descontos (93\%). Ademais, apenas $29 \%$ relataram que trabalham em alguma área relacionada à finanças ou gestão financeira.

Quando analisado apenas o número de acertos acredita-se se tratar de um bom resultado, visto que apenas 3 questões (23\%) tiveram menos de 70\% de acertos, corroborando aos achados de Potrich, Vieira e Cereta (2013) que verificaram níveis de conhecimento financeiro insatisfatório desse público.

Os resultados aqui relatados estão apresentados na Tabela 1. 
Tabela 1. Taxa de acerto das questões de conhecimento financeiro

\begin{tabular}{cccc}
\hline Var. & $\mathbf{n}$ & Média & D.P. \\
\hline Q1 & 232 & $68,97 \%$ & $46,36 \%$ \\
Q2 & 232 & $37,07 \%$ & $48,40 \%$ \\
Q3 & 232 & $71,98 \%$ & $45,01 \%$ \\
Q4 & 232 & $79,31 \%$ & $40,60 \%$ \\
Q5 & 232 & $34,05 \%$ & $47,49 \%$ \\
Q6 & 232 & $78,02 \%$ & $41,50 \%$ \\
Q7 & 232 & $82,33 \%$ & $38,23 \%$ \\
Q8 & 232 & $60,34 \%$ & $49,02 \%$ \\
Q9 & 232 & $64,66 \%$ & $47,91 \%$ \\
Q10 & 232 & $93,10 \%$ & $25,39 \%$ \\
Q11 & 232 & $97,84 \%$ & $14,55 \%$ \\
Q12 & 232 & $72,84 \%$ & $44,57 \%$ \\
Q13 & 232 & $92,24 \%$ & $26,81 \%$ \\
\hline
\end{tabular}

Fonte: Elaborado pelos autores.

Apesar disso, faz necessário uma avaliação minuciosa do instrumento de medida utilizado (questionário) com o intuito de verificar se ele mede de forma adequada o que se propõe (nível de conhecimento financeiro), inclusive para uma maior eficiência na evidenciação das propostas de verificar as diferenças entre os grupos (alunos ingressantes e concluintes e os cursos de graduação). Para isso será dado prosseguimento na estimação dos parâmetros pela TRI utilizando o modelo 2PLM.

\subsection{Mensuração do grau de conhecimento financeiro}

Inicialmente rodou-se o modelo 2PLM com todos os 13 itens do questionário, considerando apenas os questionários válidos (232). Os parâmetros estimados encontram-se na Tabela 2.

De acordo com Moreira Junior (2014) o parâmetro discriminação mostra o nível de discriminação de cada questão, distinguindo o nível de conhecimento financeiro dos indivíduos, pela variável latente $(\theta)$ medida pelo conjunto de questões respondidas, e seus valores devem orbitar entre 0 e 2 (HAMBLETON, SWAMINATHAN e ROGERS, 1991). O parâmetro dificuldade permite verificar se o item se comporta de maneira diferente entre os respondentes com o mesmo valor de traço latente (EDELEN et al., 2006; TERESI, 2006), sendo mais adequados os resultados entre -3 e +3 (MOREIRA JUNIOR, 2014). 
Tabela 2. Estimativas dos parâmetros para todas as questões

\begin{tabular}{ccccc}
\hline Questões & Discriminação & E.P. & Dificuldade & E.P. \\
\hline Q2 & 0,025935 & 0,175341 & 20,41111 & 138,0479 \\
Q4 & 0,143597 & 0,209626 & $-9,39914$ & 13,64716 \\
Q5 & 0,183279 & 0,182017 & 3,635769 & 3,634543 \\
Q6 & $0,530725 * *$ & 0,223101 & $-2,52851 * *$ & 0,999942 \\
Q8 & $0,773548 * * *$ & 0,222369 & $-0,61567 * * *$ & 0,240375 \\
Q13 & $0,901528 * * *$ & 0,348027 & $-3,10118^{* * *}$ & 0,986711 \\
Q12 & $0,964315 * * *$ & 0,257254 & $-1,21585 * * *$ & 0,294693 \\
Q1 & $0,975097 * * *$ & 0,249569 & $-0,97958 * * *$ & 0,247824 \\
Q7 & $1,168824 * * *$ & 0,314878 & $-1,6394 * * *$ & 0,340979 \\
Q9 & $1,508078 * * *$ & 0,358434 & $-0,56942 * * *$ & 0,146216 \\
Q3 & $1,524022 * * *$ & 0,357837 & $-0,87598 * * *$ & 0,172104 \\
Q11 & $2,032653 * *$ & 0,860044 & $-2,70927 * * *$ & 0,610525 \\
Q10 & 5,412427 & 3,997427 & $-1,55411 * * *$ & 0,173873 \\
\hline
\end{tabular}

Nota: E.P. indica o erro padrão dos parâmetros. ${ }^{* * *}$ Significativo a 1\%; ${ }^{* *}$ Significativo a $5 \%$.

Fonte: Elaborado pelos autores.

Após as eliminações dos itens que fugiam dos limites aceitáveis foi rodado novamente o 2MPL, os parâmetros e as estimativas estão apresentados na Tabela 3. Verifica-se que todos os parâmetros dos 7 itens se encontram dentro dos limites condizentes com a literatura (HAMBLETON, SWAMINATHAN e ROGERS, 1991; MOREIRA JUNIOR, 2014), além de apresentarem significância estatística até 5\%. Percebe-se também a existência de itens com variados níveis de dificuldade no questionário, sendo esta situação mais desejável (HARRAWAY e BARKER, 2005). Na Figura 1 apresenta-se a curva característica do teste e nela é indicado que cerca de $95 \%$ dos respondentes acertaram de 2 a 7 questões, sendo o escore máximo igual à 7 pontos, possuindo habilidade latente (theta) entre 0,6 e 6,68.

Tabela 3: Estimativas dos parâmetros e erros padrões dos itens remanescentes

\begin{tabular}{ccccc}
\hline Questões & Discriminação & E.P. & Dificuldade & E.P. \\
\hline Q6 & $0,698675 * * *$ & 0,25394 & $-1,99543 * * *$ & 0,651076 \\
Q8 & $0,8223 * * *$ & 0,238666 & $-0,58754 * * *$ & 0,227936 \\
Q1 & $0,925783 * * *$ & 0,256376 & $-1,0171 * * *$ & 0,273096 \\
Q7 & $0,947119 * * *$ & 0,306225 & $-1,89913 * * *$ & 0,500204 \\
Q12 & $0,973704 * * *$ & 0,282703 & $-1,20787 * * *$ & 0,30839 \\
Q3 & $1,721756 * * *$ & 0,459298 & $-0,82491 * * *$ & 0,163031 \\
Q9 & $1,770537 * * *$ & 0,486404 & $-0,52691 * * *$ & 0,134757 \\
\hline
\end{tabular}

Nota: ${ }^{* * *}$ Significativo a $1 \% ;{ }^{* *}$ Significativo a $5 \%$.

$\mathrm{O}$ enunciado de cada item avaliativo do conhecimento financeiro encontra-se no Apêndice.

Fonte: Elaborado pelos autores.

Em seguida, foi realizado o teste DIF (Differential Item Functioning) com intuito de investigar se a escala de conhecimento financeiro, apresentada na Tabela 3, sofre alguma variação entre os grupos da amostra (primeiro e último ano) e as variáveis de perfil binárias (gênero, se trabalha e se Revista de Educação Matemática, São Paulo, SP, v. 18, 2021, pp. 1-18 - e021053 
possui dependentes). Considerando a prerrogativa de que a função entre os grupos é não uniforme, é possível concluir a não-existência de diferenças de funções entre os grupos, nesse sentido, a função matemática estimada (os parâmetros do modelo apresentado na Tabela 3) para alunos do primeiro e último período, sejam eles homens ou mulheres e independente se trabalham com finanças ou não é igual entre os grupos. A importância dessa conclusão está no fato de que entre os grupos não se faz necessário instrumentos diferentes para medir o nível de conhecimento financeiro, sendo a escala estimada suficiente para estabelecer a classificação.

Para a classificação dos indivíduos utilizou-se critérios parecidos aos de Chen e Volpe (1998), Lusardi e Mitchell (2013), Potrich, Vieira e Ceretta (2013) os quais utilizam de percentuais, ou volumes de acertos para tal. Para isso foi considerada a seguinte classificação de conhecimento financeiro: de 0 a 4 acertos, nível baixo; 5 a 6 acertos, nível médio; 7 acertos de questões, nível alto.

Tabela 4. Distribuição dos discentes conforme nível de conhecimento financeiro e ano

\begin{tabular}{cccccccc}
\hline Conhecimento & \multirow{2}{*}{ Acertos } & \multicolumn{2}{c}{ Amostra completa } & \multicolumn{2}{c}{ Primeiro Ano } & \multicolumn{2}{c}{ Último Ano } \\
& & $\mathbf{n}$ & $\mathbf{\%}$ & $\mathbf{n}$ & $\boldsymbol{\%}$ & $\mathbf{n}$ & $\boldsymbol{\%}$ \\
\hline Baixo & De 0 a 4 & 83 & $35,78 \%$ & 55 & $41,04 \%$ & 28 & $28,57 \%$ \\
Médio & De 5 a 6 & 97 & $41,81 \%$ & 58 & $43,28 \%$ & 39 & $39,80 \%$ \\
Alto & 7 & 52 & $22,41 \%$ & 21 & $15,67 \%$ & 31 & $31,63 \%$ \\
\hline
\end{tabular}

Fonte: Elaborado pelos autores.

Dessa forma, analisando toda amostra verifica-se que a maioria apresentou conhecimentos médio (41,81\%) e baixo (35,78\%), corroborando com os achados de Potrich, Vieira e Cereta (2013). Entre alunos do primeiro e último ano (Tabela 4) percebe-se que o dobro de indivíduos do último ano foi classificado com o nível alto de conhecimento financeiro $(31,63 \%)$ em detrimento dos alunos do primeiro ano $(15,67 \%)$. Além disso, o volume de indivíduos classificados com baixo conhecimento financeiro em alunos do último ano é bem menor $(28,57 \%)$ do que àqueles alunos do primeiro ano $(41,04 \%)$.

Figura 1. Curva de Probabilidade dos Itens

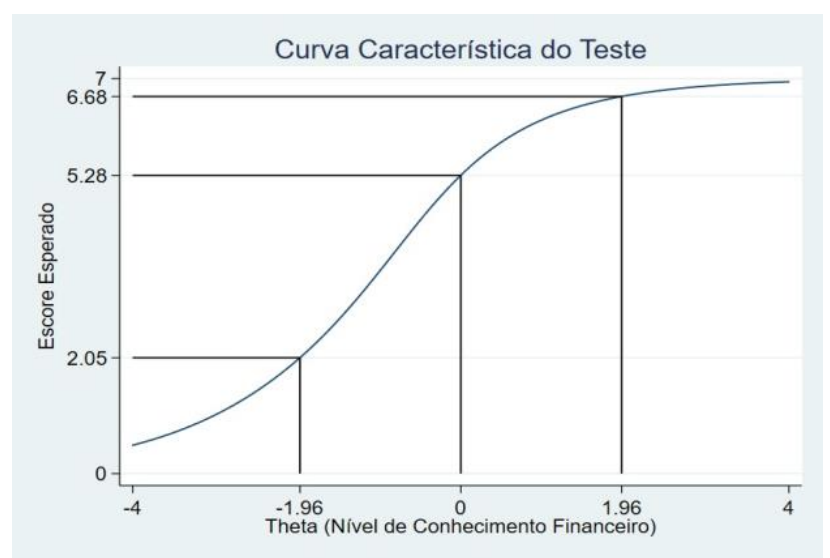

Fonte: Elaborado pelos autores. 
4.3. Diferenças no grau de conhecimento financeiro

As diferenças discutidas nessa seção são melhor visualizadas através dos gráficos apresentados na Figura 2. Pela aplicação dos testes Mann-Whitney (Z) e o teste de Kruskal-Wallis $\left(\chi^{2}\right)$ foi constatado que alunos do último ano possuem maior grau de conhecimento financeiro do que os alunos do primeiro ano $(Z=-2,786$; p-valor $<0,01)$, e que estudantes de cursos de engenharia e negócios possuem maior conhecimento financeiro que alunos de cursos de humanas $\left(\chi^{2}=21,074\right.$; $p$ valor < 0,000). Com relação aos resultados entre os períodos, o estudo de Nascimento et al. (2016) também verificou a mesma situação, apesar da fraca correlação dos resultados, em contrapartida, extrapolando entre estudantes universitários e pós-graduandos, e Nano e Polo (2016) encontraram fortes evidências de que àqueles com maior nível de escolaridade também apresentaram maior conhecimento financeiro. Com relação as diferenças entre os cursos conclui-se que aqueles que oferecem em seu currículo conteúdos relacionados a educação matemática e matemática financeira fornecem uma melhor base para o raciocínio que envolve o conhecimento financeiro.

Os testes também foram realizados para outras características da amostra, em que se verificou que homens possuem maior grau de conhecimento financeiro que mulheres $(Z=-5,207$; $p$-valor < 0,000), assim como indivíduos que se intitulam de raça branca e outros apresentaram maior nível de conhecimento financeiro, em detrimento daqueles que se declararam negros $\left(\chi^{2}=17,257\right.$; $p$-valor < 0,000).

Adicionalmente, relacionou-se o nível de conhecimento financeiro com a renda familiar e a escolaridade da mãe, pela Correlação de Spearman ( $\rho$ ) por se tratar de variáveis ordinais, constatandose que essas variáveis possuem correlação positiva. Quanto maior a renda familiar maior o nível de conhecimento financeiro $(\rho=0,19 ; \mathrm{p}$-valor $<0,00)$, sendo a maior diferença entre os alunos dos menores (até $\mathrm{R} \$ 1.900,00$ ) e maiores (acima de $\mathrm{R} \$ 5.800,00$ ) patamares. Resultado similar foi encontrado para a escolaridade da mãe $(\rho=0,23$; p-valor $<0,000)$, verificando maiores diferenças entre os níveis fundamental e superior/pós-graduação.

Esses resultados ratificam estudos como Lusardi, Mitchel e Curto (2010), Sekita (2011) e Potrich, Vieira e Cereta (2013) que encontraram nas mulheres um menor nível de conhecimento financeiro. Buscando respostas para esses achados na questão social pode-se sugerir que as mulheres apresentam uma abordagem mais passiva em relação ao dinheiro, enquanto os homens possuem uma postura ativa colocando o dinheiro como instrumento de posicionamento social (CALAMATO, 2010), onde ao buscar por meio dele uma forma de ascensão dá maior ênfase na busca por aprimorar suas habilidades em lidar com o dinheiro. 
eISSN: $2526-9062$

\section{Considerações finais}

O presente estudo teve por objetivo mensurar o nível de conhecimento financeiro de discentes de cursos de graduação de uma instituição de ensino superior pública. Optou-se por entrevistar alunos de mais de um curso independente de ofertarem em sua grade curricular disciplinas específicas de matemática, matemática financeira, gestão financeira e outros relacionados às finanças visto a importância do conhecimento financeiro para uma boa gestão das finanças pessoais de qualquer indivíduo.

Para medir o grau de conhecimento financeiro utilizou-se um questionário, o qual obteve-se um total de 232 respostas válidas, sendo estas assim utilizadas para refinar o modelo de medida pela metodologia da Teoria de Resposta ao Item com um modelo logístico unidimensional para itens dicotômicos com dois parâmetros (2PLM).

Figura 2. Diferenças no grau de conhecimento financeiro
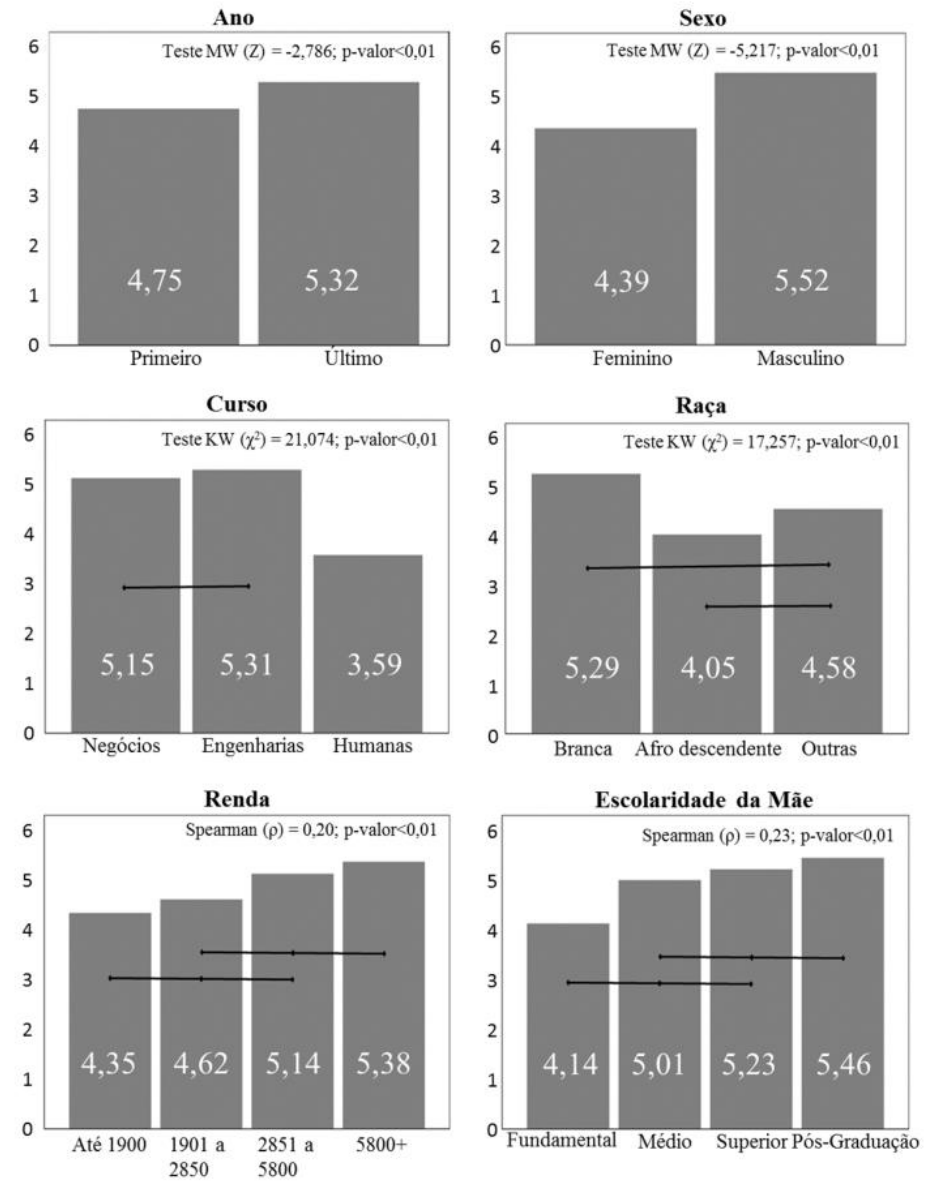

Fonte: Elaborado pelos autores.

Nota: Os traços entre as barras indicam as categorias que são diferentes após as comparações múltiplas (Teste MannWhitney com ajuste de Bonferroni) executadas após o Teste de Kruskal-Wallis $\left(\chi^{2}\right)$. Para a renda e escolaridade da mãe, além do cálculo do coeficiente de Spearman $(\rho)$, para aferição do grau da relação, também se computou o Teste KruskalWallis $\left(\chi^{2}\right)$. 
Como resultados principais foram encontrados um nível baixo $(35,78 \%)$ e médio $(41,81 \%)$ de conhecimento financeiro nos estudantes, e a existência de uma diferença de conhecimento financeiro, sendo maior em alunos do último ano (quase o dobro quando comparado aos alunos do primeiro ano) e de cursos de engenharia e negócios. Adicionalmente, encontrou-se um grau estatisticamente maior de conhecimento para indivíduos que relataram maior renda familiar e que a mãe possuía um mais alto nível de escolaridade. Ressalta-se também que os homens apresentaram um maior nível e que os indivíduos autodeclarados negros um menor nível de conhecimento financeiro.

Nesse contexto, entende-se que um melhor conhecimento das características que influenciam no aprendizado e no desenvolvimento de habilidades financeiras de um indivíduo propiciam uma melhor formulação de políticas públicas para a disseminação de formas mais eficiente de difusão do conhecimento financeiro por meio de programas educativos. Assim, estes resultados, seja a discussão da literatura ou as evidências empíricas oriundas do presente estudo, servem de suporte para incrementar o conteúdo didático da educação financeira, principalmente por meio de elucidações sobre os problemas relacionados as metodologias de disseminação deste conhecimento para diferentes públicos, os quais podem não estar sendo eficazes na sua proposta. Do ponto de vista social, educacional e mercadológico, este estudo pôde oferecer melhores evidências de como fatores relacionados ao perfil, aos diferentes cursos universitários e a vivência acadêmica podem estar associados com o aprendizado do conhecimento financeiro.

Como limitação do trabalho, cita-se a amostra, pois devido à disponibilidade e a permissão dos professores para a realização da pesquisa em sala de aula e a dificuldade em identificar os alunos de cursos e anos específicos fora das salas, esta ficou restrita. Outra limitação está na generalização dos resultados alcançados devido ao questionário: a não-disposição das questões de forma aleatória, o que eventualmente, principalmente pela aplicação em sala de aula, algumas vezes antes do início da aula, penaliza-se as últimas questões do instrumento em detrimento das primeiras tendo em vista a saturação, cansaço e pressa por parte dos alunos.

Uma abrangência de cursos pode proporcionar resultados mais robusto, no que diz respeito ao modelo proposto, bem como também expandir a análise para os outros aspectos da alfabetização financeira (atitude e comportamento financeiro), alinhando o conhecimento financeiro com estes.

Sugere-se ainda, acerca das oportunidades para condução de futuras pesquisas, com intuito de detectar a evolução do nível de conhecimento financeiro e possivelmente da alfabetização financeira em estudantes universitários, realizar um estudo longitudinal, acompanhando turmas do início até a finalização da graduação. Outra oportunidade de estudo surge na condução de pesquisas que confrontem o resultado decorrente da estimação via TRI frente à adoção de uma abordagem 
qualitativa na busca de captar eventuais motivações da ocorrência de baixos níveis de conhecimento financeiro.

\section{Referências}

Arceo-Gómez, E. O.; Villagómez, F. A. Financial literacy among Mexican high school teenagers. International Review of Economics Education, v. 24, p. 1-17, 2017.

Beal, D. J.; Delpachitra, S. B. Financial literacy among Australian university students. Economic Papers: A journal of applied economics and policy, v. 22, n. 1, p. 65-78, 2003.

Bemel, J. E.; Brower, C.; Chischillie, A.; Shepherd, J. The impact of college student financial health on other dimensions of health. American Journal of Health Promotion, v. 30, n. 4, p. 224-230, 2016.

Calamato, M. P. Learning financial literacy in the family. 2010. Master's Theses - San José State University. Disponível em: <https://scholarworks.sjsu.edu/etd theses/3849/>. Acesso em: 30/04/2021.

Chen, H.; Volpe, R. P. An analysis of personal financial literacy among college students. Financial services review, v. 7, n. 2, p. 107-128, 1998.

Cunha, F.; Heckman, J. The technology of skill formation. American Economic Review, v. 97, n. 2, p. 31-47, 2007.

Donadio, R.; Campanario, M. D. A.; Rangel, A. D. S. R. O Papel da alfabetização financeira e do cartão de crédito no endividamento dos consumidores brasileiros. Revista Brasileira de Marketing - Remark, v. 11, n. 1, p. 75-93, 2012.

Edelen, M. O.; Thissen, D.; Teresi, J. A.; Kleinman, M.; Ocepek-Welikson, K. Identification of differential item functioning using item response theory and the likelihood-based model comparison approach: application to the Mini-Mental State Examination. Medical Care, S134S142, 2006.

Embretson, S. E. The new rules of measurement. Psychological Assessment, v. 8, n. 4, p. 341-349, 1996.

Estratégia Nacional de Educação Financeira (ENEF). Quem Somos. 2021. Disponível em: <https://www.vidaedinheiro.gov.br/quemsomos/> Acesso em: 19/04/2021.

Francischetti, C. E.; Rodrigues, D. S.; Gardinal, R. R.; De Oliveira, J. A. J. Endividamento de Mercado: A necessidade de conscientização da educação financeira no Brasil. Revista de Administração do Sul do Pará (REASP)-FESAR, v. 3, n. 2, 2016.

Grohmann, A.; Kouwenberg, R.; Menkhoff, L. Childhood roots of financial literacy. Journal of Economic Psychology, v. 51, p. 114-133, 2015.

Hambleton, R. K.; Swaminathan, H.; Rogers, H. J. Fundamental of Item Response Theory. London: Sage Publications, 1991.

Harraway, J. A.; Barker, R. J. Statistics in the workplace: a survey of use by recent graduates with higher degrees. Statistics Education Research Journal, v. 4, n. 2, p. 43-58, 2005.

Huston, S. J. Measuring financial literacy. Journal of Consumer Affairs, v. 44, n. 2, p. 296-316, 2010.

Jacob, M. B. Pedagogy of Financial Education among College Students. Economics and Culture, v. 13, n. 2, p. 23-34, 2016. 
Klapper, L.; Lusardi, A.; Panos, G. A. Financial literacy and its consequences: Evidence from Russia during the financial crisis. Journal of Banking \& Finance, v. 37, n. 10, p. 3904-3923, 2013.

Knoll, M. A. Z.; Houts, C. R. The Financial Knowledge Scale: An Alication of Item Response Theory to the Assessment of Financial Literacy. Journal of Consumer Affairs, v. 46, n. 3, p. 381410, 2012.

Lusardi, A.; Mitchell, O. Older Adult Debt and Financial Frailty. Michigan Retirement Research Center Research Paper No. 291, 2013. Disponível em: 〈https://ssrn.com/abstract=23764933>. Acesso em: 30/04/2021.

Lusardi, A.; Mitchell, O. S.; Curto, V. Financial literacy among the young. Journal of consumer affairs, v. 44, n. 2, p. 358-380, 2010.

Mandell, L. The impact of financial education in high school and college on financial literacy and subsequent financial decision making. Anais in... American Economic Association Meetings, San Francisco, CA, vol. 51, 2009.

Mette, F. M. B.; De Matos, C. A. Uma Análise Bibliométrica dos Estudos em Educação Financeira no Brasil e no Mundo. Revista Interdisciplinar de Marketing, v. 5, n. 1, p. 46-63, 2016.

Moreira Junior, F. de J. Contribuições da Teoria da Resposta ao Item nas Avaliações Educacionais. Ciência e Natura, v. 36, p. 58-72, 2014.

Nano, D.; Polo, A. Academic Status Differences in Financial Literacy among Albanian University Students. EuroEconomica, v. 35, n. 1, 2016.

Nascimento, J. C. H. B. do; Macedo, M. Á. D. S.; De Siquei, J. R. M.; Bernardes, J. R. Alfabetização Financeira: um estudo por meio da aplicação da teoria de resposta ao item.

Administração: Ensino e Pesquisa, v. 17, n. 1, p. 147-175, 2016.

Norvilitis, J. M.; Merwin, M. M.; Osberg, T. M.; Roehling, P. V.; Young, P.; Kamas, M. M. Personality factors, money attitudes, financial knowledge, and credit-card debt in college students.

Journal of Applied Social Psychology, v. 36, n. 6, p. 1395-1413, 2006.

OECD/INFE. International survey of adult financial literacy competencies. 2016. Disponível em: 〈http://www.oecd.org/finance/oecd-infe-survey-adult-financial-literacy-competencies.htm>. Acesso em: 19/04/2021.

Potrich, A. C. G.; Vieira, K. M.; Campara, J. P.; Fraga, L; dos S.; Santos, L. F. D. O. Educação Financeira dos Gaúchos: proposição de uma medida e relação com as variáveis socioeconômicas e demográficas. Sociedade, Contabilidade e Gestão, v. 9, n. 3, p. 109-129, 2015.

Potrich, A. C. G.; Vieira, K. M.; Ceretta, P. S. Nível de alfabetização financeira dos estudantes universitários: afinal, o que é relevante? Revista Eletrônica de Ciência Administrativa, v. 12, n. 3, 2013.

Potrich, A. C. G.; Vieira, K. M.; Coronel, D. A.; Bender Filho, R. Financial literacy in Southern Brazil: Modeling and invariance between genders. Journal of Behavioral and Experimental Finance, 6, p. 1-12, 2015.

Potrich, A. C. G.; Vieira, K. M.; Kirch, G. Determinantes da alfabetização financeira: proposição de um modelo e análise da influência das variáveis socioeconômicas e demográficas. Anais in...

EnANPAD, XXXVIII Encontro da ANPAD, Rio de Janeiro/ RJ, 2014.

Prawitz, A. D.; Garman, E. T.; Sorhaindo, B.; O'Neill, B.; Kim, J.; Drentea, P. InCharge financial distress/financial well-being scale: Development, administration, and score interpretation. 2006. 
Reckase, M. D. Statistics for Social and Behavioral Sciences: Multidimensional Item Response Theory. Springer: New York, 2009.

Reise, S.; Ainsworth, A.; Haviland, M. Item Response Theory. Current Directions in Psychological Science, v. 14, n. 2, p. 95-101, 2005.

Scheresberg, C. B. Financial literacy and financial behavior among young adults: evidence and implications. Numeracy, v. 6, n. 2, 2013.

Sekita, S. Financial literacy and retirement planning in Japan. Journal of Pension Economics and Finance, v. 10, n. 4, p. 637-656, 2011.

Sohn, S. H.; Joo, S. H.; Grable, J. E.; Lee, S.; Kim, M. Adolescents' financial literacy: The role of financial socialization agents, financial experiences, and money attitudes in shaping financial literacy among South Korean youth. Journal of adolescence, v. 35, n. 4, p. 969-980, 2012.

Souza Fernandes, A. H. de; Candido, J. G. Educação financeira e nível do endividamento: relato de pesquisa entre os estudantes de uma instituição de ensino da cidade de São Paulo. Revista

Eletrônica Gestão e Serviços, v. 5, n. 2, p. 894-913, 2014.

Teresi, J. A. Different approaches to differential item functioning in health applications:

Advantages, disadvantages and some neglected topics. Medical care, S152-S170, 2006. 